\title{
ANÁLISE DO PROCESSO DE ADAPTAÇÃO E PADRONIZAÇÃO DA BATERIA NEUROPSICOLÓGICA LURIA-CHRISTENSEN PARA A POPULAÇÃO BRASILEIRA
}

\author{
Egídio José Romanelli'; \\ Tatiana Izabele Jaworski de Sá Riechi²; \\ Carolina Ribeiro Ambrózio ${ }^{3}$; Giovana Sato Gadens ${ }^{4 ;}$ \\ Michelle Teixeira Mitczuk ${ }^{4}$; Mirian Akiko Furutani de Oliveira ${ }^{4}$; \\ Patrícia Saddock de Sá ${ }^{4}$ Rosilene Pinto ${ }^{4}$.
}

\section{RESUMO}

Neste trabalho realiza-se uma análise do processo de adaptação e padronização da Bateria neuropsicológica Luria-Christensen para crianças, adolescentes e adultos brasileiros. Caracteriza-se por uma pesquisa motivada pela necessidade do desenvolvimento de instrumentos de avaliação diagnóstica padronizados à realidade sociocultural brasileira. A Bateria é composta por dez conjuntos de testes, e cada um deles avalia uma parte da dinâmica funcional cerebral, promovendo o mapeamento

${ }^{1}$ Professor do Departamento de Psicologia da UFPR; Doutor em Psicofisiologia pela Université de Toulouse-França. Praça Santos Andrade, 50 - $1^{\circ}$ andar - 80020-300 Curitiba - PR. E-mail: egidio@romanelli.ne.

${ }^{2}$ Professora do Departamento de Psicologia da UFPR; Mestre em Educação pela Universidade Federal do Paraná.

${ }^{3}$ Bolsista Iniciação Científica CNPq/PIBIC.

${ }^{4}$ Alunas do Curso de Psicologia da UFPR. 
qualitativo das áreas cerebrais e suas interligações, através de um exame das habilidades perceptuais, cognitivas e motoras. O trabalho de adaptação e padronização está dividido em 4 fases. A Fase 1 é dedicada ao processo tradução, mudanças e adequações das consignas, imagens e demais estímulos, elaboração de instruções de aplicação e folha de resposta, diagramação e editoração gráfica. Na Fase 2, o teste vai a campo pela primeira vez, para o grupo controle e experimental, onde após análise e devidas alterações passa para Fase 3. Nesta fase volta a campo pela segunda vez, passa novamente por análise e alterações finais; vão para a Fase 4 apenas os testes que, através da análise qualitativa e tratamento estatístico, necessitarem de nova revisão e retorno a campo. Os resultados obtidos já revelam o término da fase-piloto do trabalho, são eles: nenhum teste na Fase 1 (pré-adaptação), 03 testes na Fase 2, 07 testes na Fase 3 e 00 teste na Fase 4. A efetivação desta pesquisa vai permitir uma avaliação mais fidedigna e adequada à realidade brasileira.

Palavras-chave: 1) Avaliação Neuropsicológica; 2) Adaptação e Padronização de Testes; 3) Mapeamento das Funções Cerebrais.

\title{
ANAL YSIS OF THE PROCESS OF ADAPTATION AND STANDARDISATION OF THE LURIA-CHRISTENSEN NEURO-PSYCHOLOGICAL SET OF TESTS FOR USE BY THE BRAZILIAN POPULATION
}

\begin{abstract}
In this study, an analysis is made of the process of adapting and standardising the Luria-Christensen neuropsychological set of tests for use by Brazilian children, teenagers and adults. The process is characterised by research motivated by the need to develop diagnostic assessment instruments standardised to Brazilian social and cultural reality. The set of tests is comprised of ten
\end{abstract}


sets of tests, each one of which assesses part of the dynamic functioning of the brain, thus providing a qualitative mapping of the brain areas and their interlinking, by means of testing perceptual, cognitive and movement abilities. The task of adaptation and standardization is divided into 4 phases. Phase 1 is dedicated to the process of the translation of, changes to and adaptation of signs, pictures and other stimuli, as well as the elaboration of instructions regarding their application and answer form, and also the layout and desktop printing thereof. In Phase 2, the test is taken to the field for the first time, and applied to control and experimental groups, passing, after analysis and alterations, to Phase 3 . In Phase 3 the test is taken to the field for the second time, once more undergoing analysis and final alterations. In this way, only those tests that, after qualitative analysis and statistical treatment, still require further revision and use in the field, are subject to Phase 4 . The results obtained indicate when the pilot phase of the study can be finished, as follows: no tests in Phase 1 (pre-adaptation), 03 tests in Phase 2, 07 tests in Phase 3 and no tests in Phase 4. Once this research has been fully implemented it will be possible to obtain a more accurate assessment that is more appropriate to Brazilian reality.

Key-words: Neuropsychological Assessment; Test Adaptation and Standardisation; Brain Function Mapping.

\section{INTRODUÇÃO}

A neuropsicologia é a ciência que estuda a relação entre o cérebro e o comportamento humano. A avaliação neuropsicológica tem sido usada para estudar a organização da atividade cerebral e a relação com o comportamento e a investigação de desordens específicas cerebrais e comportamentais (Lezak, 1983).

A neuropsicologia como uma área específica de estudo tem seu desenvolvimento recente, porém está baseada em in- 
formações bastante antigas. Segundo Cunha (1993), no passado, a avaliação neuropsicológica pretendia chegar à identificação e localização de lesões cerebrais focais. Atualmente, baseia-se na localização dinâmica de funções, objetivando a investigação das funções mentais superiores, como por exemplo: a memória, a linguagem, a atenção etc. Entende a participação do cérebro como um todo, em que as áreas são interdependentes e articuladas, funcionando comparativamente a uma orquestra que depende da integração de seus componentes para realizar um concerto, conforme Luria (1981). A isto se denomina sistema funcional.

Seu principal enfoque é o desenvolvimento de uma ciência do comportamento humano baseada no funcionamento do cérebro.

Dessa forma, sabe-se que, a partir do conhecimento do desenvolvimento e funcionamento normal do cérebro, é possível a compreensão de alterações cerebrais, como no caso de disfunções cognitivas e do comportamento resultante de lesões, doenças ou desenvolvimento anormal do cérebro.

Luria, grande neuropsicólogo soviético, desenvolveu uma série de testes neuropsicológicos, formalizados por Christensen em uma bateria de avaliação neuropsicológica, dividida em dez testes que promovem um mapeamento qualitativo das áreas cerebrais e suas interligações (sistema funcional), através de um exame minucioso e sistemático de habilidades perceptuais, cognitivas e motoras (Christensen, 1978). De acordo com Gaddes, essa forma de avaliação pode diminuir a possibilidade de erros em diagnósticos e intervenções (Gaddes, 1985).

A prática da avaliação neuropsicológica caracteriza-se por uma atividade bastante complexa, que sofre interferências de diversos fatores tais como: sociais, culturais, lingüísticos, étnicos, faixa etária e características próprias de cada patologia (Kolb \& Whishaw, 1986); (Antunha, 1987). 
Sabe-se que o desenvolvimento cerebral tem características próprias a cada faixa etária; portanto, dentro desse padrão de funcionamento cerebral é importante a elaboração de provas de acordo com o processo maturacional do cérebro. Por exemplo, "quando se fala de imaturidade na infância, esta não deve ser entendida unicamente como deficiência" (Antunha, 1987: 94), devido às peculiaridades do desenvolvimento cerebral na infância. Diferentemente do adulto, o cérebro da criança está ainda em desenvolvimento, tendo características próprias que garantem uma diferenciação e especificidade de funções; para serem avaliadas, a consideração dessas funções é primordial para um diagnóstico, prognóstico e terapêutica precisa.

Antunha (1987) coloca que, devido a peculiaridades como, a) a organização e o desenvolvimento do sistema nervoso da criança; b) a dificuldade de estabelecer parâmetros de desenvolvimento devido à variabilidade entre crianças da mesma idade; e c) a estreita ligação entre o desenvolvimento físico, neurológico e a emergência progressiva de funções corticais superiores, as baterias de testes neuropsicológicos adaptados para crianças encontram-se em número bastante reduzido.

Na prática clínica, no campo da neuropsicologia, a população atendida é ampla. A avaliação neuropsicológica pode auxiliar no diagnóstico e tratamento de diversas neuropatias, problemas de desenvolvimento infantil e aprendizagem, diagnóstico diferencial, comprometimentos psiquiátricos, alterações de conduta, dependência química, entre outras. Isto indica a importância de se possuir um material fidedigno e sensível para avaliação das funções mentais superiores.

Desta forma, vê-se a importância de uma pesquisa com a finalidade de elaboração de uma bateria neuropsicológica adequada para crianças, adolescentes e adultos brasileiros.

$\mathrm{Na}$ área de avaliação, a maioria dos testes não é padronizada para a realidade brasileira e a carência de instrumentos 
de avaliação diagnóstica adequados à cultura brasileira favorece um uso equivocado e limitado dos testes estrangeiros. "Usava-se (e usa-se) os mais diversos testes, de forma indiscriminada e estereotipada, sem se considerar o sujeito, as condições de aplicação e a padronização." (Pereira \& Cardoso, 1992, p. 26). Estes testes utilizam linguagem, vocabulário e estímulos comuns ao seu país de origem, freqüentemente nos levando a diagnósticos com distorções. Outro problema é que as escalas de correção e pontuação também são padronizadas para o país de origem, o que nos leva a adotar como parâmetro de comparação uma outra realidade sociocultural.

Em vista destes fatores, além de esta bateria neuropsicológica preencher esta lacuna, ela fornece informações que os testes psicométricos não promovem, uma vez que estes se limitam a mensurar e quantificar o comportamento.

A padronização final desta bateria para as peculiaridades brasileiras produzirá uma maior eficiência do material na avaliação da integridade dos sistemas funcionais do cérebro. Diante disso, no processo de elaboração deste material de testagem levantou-se a hipótese de que, se os testes adaptados forem compreendidos pelos indivíduos que não possuam nenhum tipo de comprometimento neurológico (grupo controle) e se os achados neuropsicológicos comprovarem o diagnóstico clínico neurológico no grupo experimental, os testes então poderão ser padronizados, pois qualquer problema encontrado no desempenho do examinando durante a aplicação do teste, deverá ser associado ao sujeito e não ao teste em questão.

O objetivo principal da pesquisa é a adequação dos testes neuropsicológicos da Bateria Luria-Christensen às peculiaridades brasileiras para crianças, adolescentes e adultos, a partir da tradução para o português, realizada anteriormente por Romanelli (1994), da compilação dos Testes Neuropsicológicos de Luria feita por Christensen. Além disso, pretende-se elaborar um material pedagógico, teórico e prático, para a formação acadêmica e profissional no campo da neuropsicologia, 
através da confecção de um manual explicativo dos testes e ainda organizar informações que poderão ser úteis para outras pesquisas da área, como descrições clínicas e estudos neurofuncionais.

\section{METODOLOGIA}

A pesquisa tem seus sujeitos divididos em um grupo controle, no qual estão sujeitos sem histórico de comprometimento neurológico e um grupo experimental no qual estão sujeitos com histórico de comprometimento cerebral. O grupo controle é constituído pela população A: crianças e adolescentes sem histórico de comprometimento neurológico, entre 6 anos e 0 mês e 17 anos e 11 meses de idade, de ambos os sexos, pertencentes à rede pública e particular de ensino, da cidade de Curitiba-PR e região metropolitana e pela população C: adultos sem histórico de comprometimento neurológico entre 18 anos e 0 mês e 59 anos e 11 meses de idade, de ambos os sexos, residentes na cidade de Curitiba-PR e região metropolitana. $\mathrm{O}$ grupo experimental é constituído pela população B: crianças e adolescentes com histórico de comprometimento neurológico, entre 6 anos e 0 mês e 17 anos e 11 meses, de ambos os sexos. Essa população é composta por pacientes encaminhados pelo Setor de Pediatria do Hospital de Clínicas de Curitiba-PR ao Centro de Psicologia Aplicada (CPA) do Departamento de Psicologia da UFPR, bem como, por pacientes encaminhados de outras procedências e pela população D: adultos entre 18 anos e 0 mês e 59 anos e 11 meses com histórico de comprometimento neurológico, de ambos os sexos, residentes na cidade de Curitiba-PR e região metropolitana. Faz parte do grupo controle uma média de 50 pessoas e do grupo experimental uma média de 10 pessoas.

Como instrumentos foram utilizados: a compilação dos testes neuropsicológicos de Luria, realizada por Christensen e 
traduzida para o português por Dr. Egídio José Romanelli. Está formada por 10 testes: Funções Visuais Superiores, Organização Acústico-Motora, Funções Cutâneas Superiores e Funções Cinestésicas, Funções Motoras, Linguagem Receptiva, Linguagem Expressiva, Leitura e Escrita, Processos Mnésicos, Habilidades Aritméticas e Processos Intelectuais (Romanelli, 1994); equipamentos de filmagem para gravação e ulterior análise de algumas avaliações mais significativas; equipamentos de informática e softwares de editoração gráfica e de texto; roteiro de anamnese; caixa lúdica de triagem; testes psicométricos para auxiliar o diagnóstico neuropsicológico, que incluem sobretudo os testes de níveis de inteligência, tais como: Escala Wechsler de Inteligência para Crianças (WISC), Teste das Matrizes Progressivas RAVEN, Escala Colúmbia de Maturidade Intelectual, Teste Coletivo de Inteligência para Adultos (CIA), entre outros.

O procedimento de adaptação e padronização dos testes está dividido em quatro fases, sendo que as três primeiras são obrigatórias e a quarta fase somente é realizada para os testes que obtiveram resultados significativos para revisão indicados pelo Teste de Fisher.

A seguir apresenta-se do que se constitui cada uma das etapas do procedimento. A Fase 1 é composta de uma Pesquisa Bibliográfica $(\mathrm{PB})$, para completar informações necessárias à pré-adaptação dos testes neuropsicológicos e de uma Pré-adaptação (PA) na qual estão incluídas as seguintes atividades: revisão e elaboração das consignas e questões de testagem; seleção de figuras e material de estímulo utilizando pesquisas sobre a freqüência de aparecimento de determinadas palavras na população brasileira Pinheiro (1994) e CapoviIla (1997); elaboração de instruções de aplicação ao examinador; elaboração de folhas de resposta, de forma a facilitar a correção e análise das respostas dos examinandos; digitação e editoração gráfica; revisão do material confeccionado. 
Na Fase 2 têm-se a Coleta de dados 1 (pesquisa de campo) na qual ocorre a aplicação dos testes pré-adaptados em sujeitos das populações do grupo controle (GC1). Essa atividade é realizada por uma equipe colaboradora de acadêmicos do segundo ano de Psicologia da UFPR e por uma equipe de pesquisadores colaboradores do Laboratório de Neuropsicologia do Departamento de Psicologia da UFPR. Ocorre a aplicação dos testes pré-adaptados pelos bolsistas e equipe de pesquisadores colaboradores do Laboratório de Neuropsicologia do Departamento de Psicologia da UFPR, em sujeitos das populações do grupo experimental (GE1). Posteriormente é realizada a Análise de dados 1 (AD1), na qual se faz a avaliação qualitativa do valor funcional e da validade técnica dos estímulos sensoriais, como termos e imagens, utilizados nos testes adaptados da bateria neuropsicológica Luria-Christensen, com base nos dados coletados no grupo controle. Essa avaliação é estabelecida com base nos comentários apresentados (questões de testagem-QTs) pelos examinadores antes (QT antes) e após (QT - depois) da aplicação dos testes, com relação aos instrumentos utilizados.

Os comentários são incluídos nas seguintes categorias: adequação da tarefa propriamente dita $(\mathrm{T})$ : o comentário do examinador sugere modificações na tarefa a ser executada para que o teste seja mais eficaz na avaliação daquilo que pretende; adequação da consigna do teste $(C)$ : o comentário do examinador sugere modificações nas instruções verbais passadas ao examinando para que ele compreenda exatamente o que está sendo pedido; adequação do material de estímulo (M): o comentário do examinador sugere modificações a qualquer tipo de estímulo fornecido na execução da tarefa (estímulos verbais, visuais, táteis, cinestésicos); adequação da instrução ao examinador (I): o comentário do examinador sugere que devem ser feitas alterações que orientem e facilitem a aplicação do teste, englobando a inserção de tabelas, observa- 
ções e desenhos contidos no material do teste; adequação da folha de resposta $(F)$ : o comentário do examinador sugere alterações na estrutura das folhas de respostas para facilitar a anotação do desempenho do examinando (DRs, variáveis intervenientes durante a testagem e comentários de alterações necessárias de todo o teste); adequação da confecção do material (E): o comentário do examinador sugere alterações de erro de impressão, de montagem e ordenação de questões.

É realizada uma avaliação quantitativa que se constitui de: digitação das respostas dos sujeitos (ADa) para posterior levantamento estatístico das respostas dos sujeitos avaliados, com base nos dados coletados no grupo controle. Essa avaliação é estabelecida com base no registro das respostas apresentadas pelos sujeitos avaliados (DRs). Para cada questão do teste é estabelecido um padrão flexível de respostas aceitáveis. É realizada então uma análise comparativa entre este padrão flexível e a resposta registrada, sendo esta classificada como compatível com o padrão flexível esperado (acerto) ou incompatível (erro) (ADb).

Nesse processo são quantificados os números de acertos e erros em cada questão, e analisados estes dados, segundo o teste não paramétrico "Teste de Fisher" (Levin, 1993).

Números significativos de respostas diferentes do modelo esperado são detectados pela avaliação estatística, sendo indicado às questões que necessitam serem verificadas. $\mathrm{Na}$ aplicação do teste estatístico, as variáveis consideradas para cada questão são: o número de acertos, o número de erros, o número de acertos ideal (igual ao número total de respostas registradas na questão, o que seria igual a considerar que todas as respostas estariam corretas), o número de erros ideal (zero) e o número total de respostas registradas na questão.

Além disso, o Teste de Fisher também é aplicado no teste como um todo, analisando se o número de questões a se- 
rem verificadas é significativo em relação ao total de questões do teste. Nesse processo, as variáveis consideradas são: o número de questões $\mathrm{OK}$, o número de questões com indicação para serem verificadas, o valor ideal para o número de questões OK (igual ao número total de questões, o que seria igual a considerar que todas as questões estariam adequadas), o valor ideal para o número de questões a serem verificadas (zero) e o número total de questões (ADc). Concomitantemente a este processo descrito, faz-se a descrição dos resultados (ADd), bem como a discussão dos mesmos (Ade).

Com os sujeitos do grupo experimental é realizado um cruzamento de dados com avaliações neuropsicológicas e diagnósticos clínicos neurológicos

No procedimento de Adaptação 1 (A1), incluem-se as seguintes atividades, com base nos dados obtidos na Análise de dados 1 (AD1) que indicam as possíveis falhas no instrumento favorecendo a sua correção: alteração de figuras e material de estímulo; alteração de instruções de aplicação ao examinador; alteração das folhas de resposta; digitação e editoração gráfica; revisão final do material da adaptação piloto.

Na Fase 3 repetem-se as etapas da fase 2. A Padronização piloto $(P)$ da Fase 3 é feita com base nos dados obtidos em (AD2) que indicam as possíveis falhas no instrumento favorecendo a sua correção. Realizam-se: alteração de figuras e material de estímulo; alteração de instruções de aplicação ao examinador; alteração das folhas de resposta; digitação e editoração gráfica e a revisão final do material da padronização piloto.

A Fase 4 somente será realizada para os testes que obtiveram resultados significativos para revisão indicados pelo Teste de Fisher; seguindo o mesmo procedimento da fase anterior. 
Egídio J. Romanelli; Tatiana I. J. de Sá Reichi e col.

\section{RESULTADOS}

Os resultados obtidos na pesquisa até o presente momento são: nenhum teste na fase 1,07 testes na fase 2, 07 teste na fase 3 e nenhum teste na fase 4 . Abaixo se apresenta um quadro comparativo, mostrando a evolução dos testes durante os três anos de pesquisa.

Quadro comparativo 1: evolução dos testes ao longo de três anos de pesquisa.

\begin{tabular}{|c|c|c|c|c|c|}
\hline & Fase 1 & Fase 2 & Fase 3 & Padronização & Fase 4 \\
\hline 1998 & $\begin{array}{l}\Rightarrow F V S \\
\Rightarrow \text { OAM } \\
\Rightarrow \text { LEsc } \\
\Rightarrow \text { HÁ } \\
\Rightarrow \text { FM } \\
\Rightarrow \text { LExp } \\
\Rightarrow P I\end{array}$ & $\begin{array}{l}\Rightarrow \text { FCSFC } \\
\Rightarrow P M\end{array}$ & $\Rightarrow L R$ & & \\
\hline 1999 & $\begin{array}{l}\Rightarrow \text { LEsc } \\
\Rightarrow H A \\
\Rightarrow F M \\
\Rightarrow \text { LExp }\end{array}$ & $\begin{array}{l}\Rightarrow F V S \\
\Rightarrow O A M \\
\Rightarrow P I\end{array}$ & $\Rightarrow$ FCSFC & $\begin{array}{l}\Rightarrow \mathrm{LR} \\
\Rightarrow \mathrm{PM}\end{array}$ & \\
\hline 2000 & & $\begin{array}{l}\Rightarrow \text { LExp } \\
\Rightarrow \text { FM } \\
\Rightarrow \text { LEsc }\end{array}$ & $\begin{array}{l}\Rightarrow P I \\
\Rightarrow F C S F C\end{array}$ & $\begin{array}{l}\Rightarrow H A \\
\Rightarrow F V S \\
\Rightarrow O A M \\
\Rightarrow L R \\
\Rightarrow P M\end{array}$ & \\
\hline
\end{tabular}

\section{Legenda do quadro:}

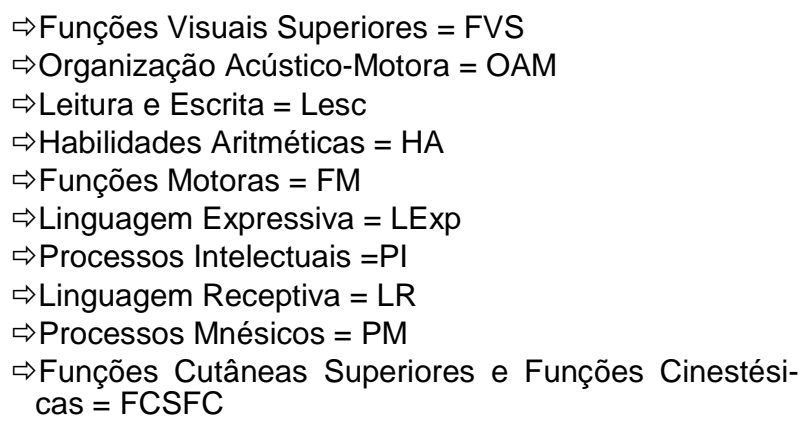




\section{DISCUSSÃO}

O processo de adaptação e padronização de um teste para a realidade sociocultural de um país é algo que demanda tempo e rigor nas etapas pelas quais ele passa. A adaptação e a padronização da Bateria Neuropsicológica Luria-Christensen para a realidade brasileira vêm seguindo os critérios existentes na literatura científica para a sua construção.

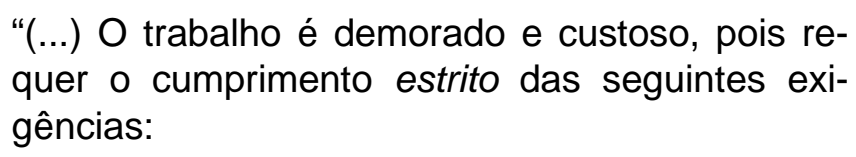

“(...) O trabalho é demorado e custoso, pois requer o cumprimento estrito das seguintes exigências:

- definição funcional do atributo visado (apoiada no exame da literatura técnica correspondente e em observações práticas);

- especificação dos fins a que um instrumento quer servir;

- planejamento da sua forma geral (tipo, conteúdo, formato e material de apoio) e do seu tempo de aplicação;

- preparação do roteiro básico para sua montagem;

- elaboração da versão preliminar do instrumento;

- estudo piloto do funcionamento da versão preliminar em uma pequena amostra da população alvo;

- análise qualitativa e quantitativa dos resultados desse estudo;

- reajuste ou melhorias no instrumento, em função do observado no estudo-piloto;

- montagem e aplicação de versões experimentais sucessivas que vão sendo aperfeiçoadas em 
conseqüência da análise do funcionamento de cada uma; em fases que se repetem. Até se conseguir uma versão aceitável do instrumento, reexaminam-se todos os seus elementos: desde a adequação a população alvo e a integração das partes que o compõem, ao funcionamento das instruções, o formato e as condições de aplicação da prova e o seu grau de precisão e qualidade, bem como o nível de dificuldade e poder de discriminação de cada questão;

- montagem da forma final do teste; (...)" (Medeiros, 1999: 141).

A pesquisa de campo é extremamente importante em todo o processo, uma vez que oferece subsídios que permitem eliminar ao máximo as variáveis intervenientes na testagem e resguardar a sensibilidade do material na detecção das habilidades a serem avaliadas.

Pode-se observar que algumas alterações propostas foram significativas, uma vez que se tem procurado o maior rigor possível no que diz respeito à validade técnica e funcional dos estímulos verbais, visuais e cenestésicos específicos para as peculiaridades brasileiras.

A pesquisa tem se preocupado com a qualidade do material; devido a isso, algumas alterações se fizeram necessárias. Por exemplo, no teste de Funções Visuais Superiores, houve a troca de figuras estilizadas por fotos, uma vez que estas permitem uma melhor identificação do que é solicitado na tarefa e provavelmente os erros que ocorrem na tarefa são do sujeito e não da tarefa em questão. No teste de Organização AcústicoMotora, houve a troca do instrumento musical de aplicação xilofone para um teclado, eliminando uma das variáveis intervenientes do instrumento anterior que era a intensidade com que o som era emitido. 
Algumas alterações na forma original do teste mostraramse fundamentais para melhorar a qualidade dos testes e adaptá-los para o ensino escolar brasileiro, não perdendo de vista o objetivo original dos testes. Estas alterações ocorreram nos testes de Habilidades Aritméticas e Leitura e Escrita que, diferentemente dos demais, estão atrelados à questão da escolaridade. Estes testes foram divididos para a população conforme a escolaridade e não conforme a faixa etária, na tentativa de controlar a variável escolaridade.

Com o objetivo de melhorar as consignas e o entendimento das tarefas pelos examinandos, optou-se pela utilização de dois tipos de comando: frases semidiretivas e frases explicativas. As frases semidiretivas como por exemplo: "Quero que você me diga...", são usadas nos testes de adultos ou de um nível superior de escolaridade. Há uma mescla de frases semidiretivas e frases explicativas como por exemplo: "Vou mostrar para você... e quero que você...", quando se trata de um teste elaborado para crianças ou para um nível de escolaridade inferior, a fim de que haja a compreensão do comando. As frases diretivas como, por exemplo: "Diga...", que eram usadas no início da pesquisa foram "abandonadas", pois se constatou que elas dificultavam a compreensão da tarefa.

A pesquisa tem priorizado a modificação dos testes que se encontravam em fase 1 procurando passá-los para a fase 2. Isto se deve à necessidade de um material que possa ser usado nas avaliações neuropsicológicas realizadas nos demais projetos desenvolvidos no Laboratório de Neuropsicologia da UFPR. Assim, pela análise de dados, é possível perceber um avanço dos seguintes testes: Leitura e Escrita, Habilidades Aritméticas, Linguagem Expressiva e Funções Motoras. Desta forma, a bateria já apresenta os 10 testes pré-adaptados, que apresentam no momento consignas, instruções, cartões e outros materiais de estímulo mais próximos à realidade sociocultural nacional, o que favorece o uso destes testes na popu- 
lação brasileira garantindo uma maior fidedignidade do material utilizado.

Desta maneira, desenvolver um material que se adapte à realidade cultural do Brasil tem sua importância fundamentada na necessidade de se ter como parâmetro de execução a própria população a ser avaliada.

\section{CONCLUSÃO}

O processo de padronização de qualquer material é bastante complexo. A metodologia varia conforme o material e seus objetivos. Após anos em que esta pesquisa vem se realizando, com o crescimento metodológico hoje alcançado, principalmente com os apoios da estatística, informática e designer, pode-se visualizar o término da primeira fase do projeto, enquanto piloto, e se organizar para a segunda parte, que seria a aplicação do material padronizado para uma população significativamente maior e, conseqüentemente sua publicação. Uma vez que a proposta metodológica utilizada neste trabalho tem apresentado resultados bastante satisfatórios e consistentes.

Dois fatores mostram-se fundamentais para um processo de avaliação satisfatório. Um é o objetivo, o que se quer avaliar, e o outro é a qualidade do instrumento. Ao escolher um instrumento de avaliação, o psicólogo deve confiar neste.

Para que se tenha uma padronização rigorosa da pesquisa, deve-se levar em conta a adequação das consignas dos testes, condições de aplicação, preparo do aplicador, devendo haver uniformidade de aplicação, apesar de examinadores diferentes, considerando o tempo de aplicação, a ordem e a forma dos itens, bem como o material a ser utilizado.

Estabelecer como objetivo o desenvolvimento de um material de testagem que seja isento de variáveis que interfiram na aplicação do mesmo, é estabelecer um objetivo metodologi- 
camente impossível de ser alcançado. Entretanto, o de- senvolvimento de materiais que possibilitem um maior grau de segurança em procedimentos de avaliação, outorga ao examinador e aos profissionais que precisam dos resultados de sua avaliação, instrumental mais claro para o desenvolvimento de programas de intervenção pós-avaliação.

As variáveis que interferem na análise de resultados englobam um espectro muito amplo, sendo que um dos aspectos que mais interferem em testagens é a inadequação de material, que traz em sua estruturação peculiaridades que lhe são específicas.

A etapa de coleta de dados apresenta-se como a etapa de feedback acerca do material em processo de padronização, possibilitando desta forma aprimorar qualquer uma das categorias de modificações do material levado a campo; fornece dados relevantes que podem ser estendidos a outros testes em fase de pré-adaptação ou adaptação.

Diante disso, observa-se a necessidade da continuidade do processo de adaptação e padronização, visto que esse trabalho permite concluir a validade funcional e técnica do instrumento, bateria Neuropsicológica Luria-Christensen, adaptada às peculiaridades da realidade sociocultural brasileira para crianças, adolescentes e adultos. Com a realização desta pesquisa, pôde-se verificar a importância de se obterem dados teoricamente mais coerentes e tecnicamente mais precisos, que fornecem subsídios para outras pesquisas.

\section{REFERÊNCIAS BIBLIOGRÁFICAS}

Antunha, E. L. (1987). Investigação neuropsicológica na infância. Boletim de Psicologia da Sociedade de Psicologia de São Paulo, XXXVII (87), 80-102. 
Capovilla, F.C. E Capovilla, A.G.S. (1997). Desenvolvimento lingüístico na criança dos dois aos 6 anos: Tradução e Estandardização do Peabody Picture Vocabulary Test de Dunn \& Dunn e da Language Development Survey de Rescorla. Ciência Cognitiva: teoria, pesquisa e aplicação, 1(1), 249-300.

Cunha, J. A. (1993). Psicodiagnóstico-R. Porto Alegre: Artes Médicas.

Christensen, A. (1978). Los Testes Neuropsicológicos de Luria. Madri: Visor Libros.

Gaddes, W. (1985). Learning disabilities and brain function: a neuropsychological approach. New York: Springer-Verlag.

Kolb, B \& Whishaw, I. (1986). Fundamentos de Neuropsicologia Humana. Barcelona: Labor.

Levin, J. (1993). Estatística Aplicada às ciências humanas. São Paulo: Harbra.

Lezak, M. D. (1995). Neuropsychological Assessment. 3. ed. New York: Oxford University Press.

Luria, A. R. (1981). Fundamentos de Neuropsicologia. São Paulo: EPU.

Medeiros, E. B. (1999). Medidas Psico \& Lógicas: introdução à psicometria. Rio de Janeiro: Ediouro.

Mitczuk, M. T.; Pinto, R. \& Sampaio, V.M. (1999). Avaliação Neuropsicológica: contribuições teórico-práticas para a psicologia e áreas afins. Relatório de pesquisa. Não publicado, Universidade Federal do Paraná, Curitiba.

Pereira, A. M. P. B. \& Cardoso, J. M. M. (1992). Testes Psicológicos: o que pretendemos. Documenta CRP- 08, 2, 20-41.

Pinheiro, A. M. (1994). Leitura e Escrita: uma abordagem Cognitiva. São Paulo: Editorial Psy II.

Romanelli, E. J. (1994). Tradução da bateria de Testes Neuropsicológicos de Luria. Relatório de pesquisa PIBIC/CNPQ. Não publicado, Universidade Federal do Paraná, Curitiba. 